\title{
Erratum to: Single projection Kaczmarz extended algorithms
}

\author{
Stefania Petra ${ }^{1}$ Constantin Popa ${ }^{2}$
}

Published online: 14 August 2017

(C) Springer Science+Business Media, LLC 2017

Erratum to: Numerical Algorithms, 73(3)(2016), 791-806 https://doi.org/10.1007/s11075-016-0118-7

\section{Comments and notations}

We made corrections only on section "Convergence analysis" of the original paper. We will refer to the equations, results, and references from the original paper by adding the sign $(*)$. Else, these are related to the Erratum version of section "Convergence analysis".

\section{Erratum - convergence analysis}

In order to prove the convergence of the two algorithms MREK and ACEK, we next examine how the distance to any fixed least-squares solution changes. We followed the general ideas of considering projection based algorithms together with relaxation

The online version of the original article can be found at doi:10.1007/s11075-016-0118-7.

Constantin Popa

cpopa@univ-ovidius.ro

Stefania Petra

petra@math.uni-heidelberg.de

1 Mathematical Imaging Group, University of Heidelberg, 69115 Heidelberg, Germany

2 Faculty of Mathematics and Informatics, OVIDIUS University of Constanta, Blvd. Mamaia 124, 900527 Constanţa, Romania 
parameters, as in the original paper by Kaczmarz [*3] and further developments, see e.g. the monographs [*1], [*4].

To this end, we denote by $x_{*}^{k}=P_{H_{i_{k}}}^{\omega}\left(x^{k-1}\right)$, where $H_{i_{k}}$ is the unperturbed hyperplane from $(* 18)$, given by

$$
x_{*}^{k}=x^{k-1}-\omega \frac{\left\langle A_{i_{k}}, x^{k-1}\right\rangle-b_{i_{k}}}{\left\|A_{i_{k}}\right\|^{2}} A_{i_{k}} .
$$

Proposition 1 For any $x \in L S S(A ; \hat{b})$ and for all $k \in \mathbb{N}$, we have for every iterate $x^{k}$ generated by the algorithm MREK or ACEK, respectively and for any $i_{k} \in[\mathrm{m}]$

(i)

$$
\left\langle x_{*}^{k}-x, \omega \gamma_{i_{k}}\right\rangle=(1-\omega)\left\langle\frac{\left\langle A_{i_{k}}, x^{k-1}-x\right\rangle}{\left\|A_{i_{k}}\right\|^{2}} A_{i_{k}}, \omega \gamma_{i_{k}}\right\rangle,
$$

(ii)

$$
\begin{gathered}
\left\|x^{k}-x\right\|^{2}=\left\|x^{k-1}-x\right\|^{2}+\frac{\omega^{3}(2-\omega)+(1-\omega)^{2} \omega^{2}}{\omega(2-\omega)}\left\|\gamma_{i_{k}}\right\|^{2} \\
-\left\|\sqrt{\omega(2-\omega)} \frac{\left\langle A_{i_{k}}, x^{k-1}-x\right\rangle}{\left\|A_{i_{k}}\right\|^{2}} A_{i_{k}}-\frac{1-\omega}{\sqrt{\omega(2-\omega)}} \omega \gamma_{i_{k}}\right\|^{2},
\end{gathered}
$$

(iii)

$$
\left\|x^{k}-x\right\|^{2} \leq\left\|x^{k-1}-x\right\|^{2}+\frac{\omega^{3}(2-\omega)+(1-\omega)^{2} \omega^{2}}{\omega(2-\omega)}\left\|\gamma_{i_{k}}\right\|^{2},
$$

with $\gamma_{i_{k}}$ from $(* 19)$.

Proof (i) Choose $x \in L S S(A ; \hat{b})$ arbitrarily. Then $A x=b$ and, in particular, $x \in H_{i_{k}}$. If $S_{i_{k}}=\left\{x:\left\langle A_{i_{k}}, x\right\rangle=0\right\}$ is the vector subspace parallel with $H_{i_{k}}$ and

$$
P_{S_{i_{k}}}^{\omega}(z)=z-\omega \frac{\left\langle A_{i_{k}}, z\right\rangle}{\left\|A_{i_{k}}\right\|^{2}} A_{i_{k}}, \quad P_{S_{i_{k}}}(z)=z-\frac{\left\langle A_{i_{k}}, z\right\rangle}{\left\|A_{i_{k}}\right\|^{2}} A_{i_{k}}
$$

are the corresponding projections operators, from (1) and (*19) it results

$$
x_{*}^{k}-x=P_{S_{i_{k}}}^{\omega}\left(x^{k-1}-x\right)=P_{S_{i_{k}}}\left(x^{k-1}-x\right)+(1-\omega) \frac{\left\langle A_{i_{k}}, x^{k-1}-x\right\rangle}{\left\|A_{i_{k}}\right\|^{2}} A_{i_{k}}
$$

and that the vectors $P_{S_{i_{k}}}\left(x^{k-1}-x\right)$ and $\gamma_{i_{k}}$ are orthogonal. These give us the equality (2). 
(ii) First of all, because the application $P_{S_{i_{k}}}^{\omega}$ has similar properties with $\varphi_{j}^{\alpha}$ (see (*38)), from Lemma *3 (see $(* 40)$ ) applied to $P_{S_{i_{k}}}^{\omega}$ and $P_{S_{i_{k}}}$ we get (by also using the fact that the projection $P_{S_{i_{k}}}$ is an idempotent operator)

$$
\begin{aligned}
\left\|x_{*}^{k}-x\right\|^{2}= & \left\|P_{S_{i_{k}}}^{\omega}\left(x^{k-1}-x\right)\right\|^{2} \\
= & \omega(2-\omega)\left(\left\|P_{S_{i_{k}}}\left(x^{k-1}-x\right)\right\|^{2}-\left\|x^{k-1}-x\right\|^{2}\right) \\
& +\left\|x^{k-1}-x\right\|^{2} \\
= & \omega(2-\omega)\left\langle P_{S_{i_{k}}}\left(x^{k-1}-x\right), x^{k-1}-x\right\rangle \\
& +(1-\omega(2-\omega))\left\|x^{k-1}-x\right\|^{2} \\
= & \left\|x^{k-1}-x\right\|^{2}-\omega(2-\omega) \frac{\left\langle A_{i_{k}}, x^{k-1}-x\right\rangle^{2}}{\left\|A_{i_{k}}\right\|^{2}} .
\end{aligned}
$$

Then, from (*16), (1), (6) and (2) we successively have

$$
\begin{gathered}
\left\|x^{k}-x\right\|^{2}=\left\|\left(x_{*}^{k}-x\right)+\omega \gamma_{i_{k}}\right\|^{2}=\left\|x_{*}^{k}-x\right\|^{2}+\omega^{2}\left\|\gamma_{i_{k}}\right\|^{2}+2\left\langle x_{*}^{k}-x, \omega \gamma_{i_{k}}\right\rangle \\
=\left\|x^{k-1}-x\right\|^{2}-\omega(2-\omega) \frac{\left\langle A_{i_{k}}, x^{k-1}-x\right\rangle^{2}}{\left\|A_{i_{k}}\right\|^{2}}+\omega^{2}\left\|\gamma_{i_{k}}\right\|^{2} \\
+2(1-\omega)\left\langle\frac{\left\langle A_{i_{k}}, x^{k-1}-x\right\rangle}{\left\|A_{i_{k}}\right\|^{2}} A_{i_{k}}, \omega \gamma_{i_{k}}\right\rangle .
\end{gathered}
$$

Now, for simplifying the presentation, we will introduce the notations

$$
D=\frac{\left\langle A_{i_{k}}, x^{k-1}-x\right\rangle}{\left\|A_{i_{k}}\right\|^{2}} A_{i_{k}}, \quad B=\omega \gamma_{i_{k}},
$$

which gives us

$$
\|D\|^{2}=\frac{\left\langle A_{i_{k}}, x^{k-1}-x\right\rangle^{2}}{\left\|A_{i_{k}}\right\|^{2}}, \quad\|B\|^{2}=\omega^{2}\left\|\gamma_{i_{k}}\right\|^{2} .
$$

By using these notations we compute as follows (see the second and fourth terms in the last sum from (7))

$$
\begin{aligned}
- & \omega(2-\omega)\|D\|^{2}+2(1-\omega)\langle D, B\rangle \\
= & -\left[\langle\sqrt{\omega(2-\omega)} D, \sqrt{\omega(2-\omega)} D\rangle-2\left\langle\sqrt{\omega(2-\omega)} D, \frac{1-\omega}{\sqrt{\omega(2-\omega)}} B\right\rangle\right] \\
& +\left[\left\langle\frac{1-\omega}{\sqrt{\omega(2-\omega)}} B, \frac{1-\omega}{\sqrt{\omega(2-\omega)}} B\right\rangle-\left\langle\frac{1-\omega}{\sqrt{\omega(2-\omega)}} B, \frac{1-\omega}{\sqrt{\omega(2-\omega)}} B\right\rangle\right] \\
= & -\left\|\sqrt{\omega(2-\omega)} D-\frac{1-\omega}{\sqrt{\omega(2-\omega)}} B\right\|^{2}+\frac{(1-\omega)^{2}}{\omega(2-\omega)}\|B\|^{2} .
\end{aligned}
$$

Then, (3) follows from (7) and (10).

(iii) It results directly from (3) and the proof is complete.

The next Lemma is a special case of Lemma 3.1 in [*2]. The corresponding simplified proof is included for completeness. 
Lemma 1 Let $\left(\alpha_{k}\right)_{k \in \mathbb{N}} \in \ell_{+}$and $\left(\beta_{k}\right)_{k \in \mathbb{N}} \in \ell_{+}$be two nonnegative sequences, and $\left(\varepsilon_{k}\right)_{k \in \mathbb{N}} \in \ell_{+} \cap \ell^{1}$ satisfying

$$
\alpha_{k+1}=\alpha_{k}-\beta_{k}+\varepsilon_{k} .
$$

Then the following statements hold true.

(i) $\left(\beta_{k}\right)_{k \in \mathbb{N}} \in \ell^{1}$. In particular $\left(\beta_{k}\right)_{k \in \mathbb{N}} \in \ell_{c_{0}}$,

(ii) $\left(\alpha_{k}\right)_{k \in \mathbb{N}}$ converges.

Proof (i) From (11), we have $\beta_{k}=\alpha_{k}-\alpha_{k+1}+\varepsilon_{k}$. Furthermore,

$$
\sum_{k=0}^{n} \beta_{k}=\sum_{k=0}^{n}\left(\alpha_{k}-\alpha_{k+1}\right)+\sum_{k=0}^{n} \varepsilon_{k}=\alpha_{0}-\alpha_{n+1}+\sum_{k=0}^{n} \varepsilon_{k}<\alpha_{0}+\sum_{k=0}^{n} \varepsilon_{k},
$$

which yields $\sum_{k \in \mathbb{N}} \beta_{k}<\alpha_{0}+\sum_{k \in \mathbb{N}} \varepsilon_{k}<+\infty$. Hence $\left(\beta_{k}\right)_{k \in \mathbb{N}} \in \ell^{1}$. Now $\ell^{1} \subset \ell_{c_{0}}$, shows (i).

(ii) Now, both $\left(\varepsilon_{k}\right)_{k \in \mathbb{N}} \in \ell_{c_{0}}$ and $\left(\beta_{k}\right)_{k \in \mathbb{N}} \in \ell_{c_{0}}$. By (11),

$$
\left|\alpha_{k+1}-\alpha_{k}\right|=\left|\varepsilon_{k}-\beta_{k}\right| \leq\left|\varepsilon_{k}\right|+\left|\beta_{k}\right|=\varepsilon_{k}+\beta_{k},
$$

with $\left(\varepsilon_{k}+\beta_{k}\right)_{k \in \mathbb{N}} \in \ell^{1}$. This shows that $\left(\alpha_{k}\right)_{k \in \mathbb{N}}$ is a Cauchy sequence. ${ }^{1}$ Since $\left(\alpha_{k}\right)_{k \in \mathbb{N}} \in \ell_{+} \subset \mathbb{R}$ it also converges.

We are now ready to prove convergence of MREK.

Theorem 1 Let $\alpha, \omega \in(0,2)$. The sequence $\left(x^{k}\right)_{k \in \mathbb{N}}$ generated by the MREK, algorithm converges to a least-squares solution in $\operatorname{LS} S(A ; \hat{b})$, for any starting vector $x^{0} \in \mathbb{R}^{n}$.

Proof We split the proof into two parts, showing convergence of $\left(x^{k}\right)_{k \in \mathbb{N}}$, and convergence to a point in $\operatorname{LSS}(A ; \hat{b})$, respectively.

(i) Choose any $x \in \operatorname{LSS}(A ; \hat{b})$ and set

$$
\begin{gathered}
\alpha_{k+1}=\left\|x^{k}-x\right\|^{2}, \quad \varepsilon_{k}=\frac{\omega^{3}(2-\omega)+(1-\omega)^{2} \omega^{2}}{\omega(2-\omega)}\left\|\gamma_{i_{k}}\right\|^{2}, \\
\beta_{k}=\left\|\sqrt{\omega(2-\omega)} \frac{\left\langle A_{i_{k}}, x^{k-1}\right\rangle-x}{\left\|A_{i_{k}}\right\|^{2}} A_{i_{k}}-\frac{1-\omega}{\sqrt{\omega(2-\omega)}} \omega \gamma_{i_{k}}\right\|^{2} .
\end{gathered}
$$

The above Lemma 1 asserts convergence of $\left(\alpha_{k}\right)_{k \in \mathbb{N}}$ and $\left(\beta_{k}\right)_{k \in \mathbb{N}} \in \ell^{1}$, in view of $\varepsilon_{k} \in \ell^{1}$, due to Lemma $* 2$ (ii) and Proposition 1 (ii) respectively. From (*16)

\footnotetext{
${ }^{1}$ An arbitrary sequence $\left(y^{k}\right)_{k \in \mathbb{N}}$ is Cauchy, if $\left\|y^{k+1}-y^{k}\right\| \leq a_{k}$ holds for all $k \in \mathbb{N}$ and $\left(a_{k}\right)_{k \in \mathbb{N}} \in \ell^{1} \cap \ell_{+}$ arbitrary. Indeed, $\left\|y^{m+k}-y^{m}\right\|=\left\|\sum_{j=m}^{m+k-1}\left(y^{j+1}-y^{j}\right)\right\| \leq \sum_{j=m}^{m+k-1}\left\|y^{j+1}-y^{j}\right\| \leq \sum_{j=m}^{m+k-1} a_{j}=$ $s_{m+k-1}-s_{m-1}$, with $s_{n}:=\sum_{j=1}^{n} a_{j}$. Now $\left(s_{n}\right)_{k \in \mathbb{N}}$ is Cauchy since it converges due to $\left(a_{k}\right)_{k \in \mathbb{N}} \in \ell^{1}$.
} 
and by using again the notations (8)-(9) we get (by also using the elementary inequality $\left.(a+b)^{2} \leq 2\left(a^{2}+b^{2}\right)\right)$

$$
\begin{aligned}
& \left\|x^{k}-x^{k-1}\right\|^{2}=\omega^{2}\left\|-\frac{\left\langle A_{i_{k}}, x^{k-1}\right\rangle-b_{i_{k}}}{\left\|A_{i_{k}}\right\|^{2}} A_{i_{k}}+\gamma_{i_{k}}\right\|^{2}=\omega^{2}\left\|D-\frac{1}{\omega} B\right\|^{2} \\
& =\frac{\omega^{2}}{\omega(2-\omega)}\left\|\sqrt{\omega(2-\omega)} D-\frac{\sqrt{\omega(2-\omega)}}{\omega} B\right\|^{2} \\
& =\frac{\omega}{2-\omega}\left\|\left(\sqrt{\omega(2-\omega)} D-\frac{1-\omega}{\sqrt{\omega(2-\omega)}} B\right)+\left(\frac{1-\omega}{\sqrt{\omega(2-\omega)}}-\frac{\sqrt{\omega(2-\omega)}}{\omega}\right) B\right\|^{2} \\
& \leq 2 \frac{\omega}{2-\omega}\left(\left\|\sqrt{\omega(2-\omega)} D-\frac{1-\omega}{\sqrt{\omega(2-\omega)}} B\right\|^{2}+\frac{1}{\omega(2-\omega)}\|B\|^{2}\right) \\
& \leq \frac{2 \omega}{2-\omega} \beta_{k}+\frac{2 \omega}{(2-\omega)\left(\omega+(1-\omega)^{2}\right)} \varepsilon_{k} .
\end{aligned}
$$

Now $\left(\frac{2 \omega}{2-\omega} \beta_{k}+\frac{2 \omega}{(2-\omega)\left(\omega+(1-\omega)^{2}\right)} \varepsilon_{k}\right)_{k \in \mathbb{N}} \in \ell^{1}$ implies that $\left(x^{k}\right)_{k \in \mathbb{N}}$ is a Cauchy sequence $^{2}$ and converges as well: assume that $x^{k} \rightarrow \bar{x}$. In particular, using again (*16), $\left\|x^{k}-x^{k-1}\right\| \rightarrow 0$ and $\gamma_{i_{k}} \rightarrow 0$ (see e.g. $(* 33)$ ) we obtain

$$
\frac{\left(\left\langle A_{i_{k}}, x^{k-1}\right\rangle-\hat{b}_{i_{k}}^{k}\right)^{2}}{\left\|A_{i_{k}}\right\|^{2}} \rightarrow 0 .
$$

(ii) We show that $\bar{x} \in \operatorname{LSS}(A, \hat{b})$. Fix any $i \in[m]$. Due to the particular choice of $i_{k}$ in $(* 21)$, we have

$$
\begin{aligned}
\left|\left\langle A_{i}, x^{k-1}\right\rangle-b_{i}\right|-\left|r_{i}-y_{i}^{k}\right| & \leq\left|\left\langle A_{i}, x^{k-1}\right\rangle-b_{i}-\left(r_{i}-y_{i}^{k}\right)\right| \\
& =\left|\left\langle A_{i}, x^{k-1}\right\rangle-b_{i}^{k}\right| \\
& \stackrel{(* 24)}{\leq}\left|\left\langle A_{i_{k}}, x^{k-1}\right\rangle-b_{i_{k}}^{k}\right| .
\end{aligned}
$$

Thus $\left|\left\langle A_{i}, x^{k-1}\right\rangle-b_{i}\right| \rightarrow 0$, due to $\left|r_{i}-y_{i}^{k}\right| \rightarrow 0$ by Lemma $* 2$ (iii) and (14), respectively. Summarizing, we get $\lim _{k \rightarrow \infty}\left\|A x^{k-1}-b\right\|=0=\|A \bar{x}-b\|$. Thus, $\bar{x} \in S(A ; b)=\operatorname{LSS}(A, \hat{b})$.

The main result concerning convergence of ACEK is stated next.

Theorem 2 The sequence $\left(x^{k}\right)_{k \in \mathbb{N}}$ generated by ACEK, converges to a least-squares solution in $\operatorname{LS} S(A ; \hat{b})$, for any starting vector $x^{0} \in \mathbb{R}^{n}$.

Proof Choose any $x \in \operatorname{LSS}(A ; \hat{b})$ and set $\alpha_{k+1}, \quad \beta_{k}, \quad \varepsilon_{k}$ as in (12). The proof of convergence $x^{k} \rightarrow \bar{x}$ is identically to the first part of the proof of Theorem 1, with the only difference that we have $\left(\varepsilon_{k}\right)_{k \in \mathbb{N}} \in \ell^{1}$ due to Lemma $* 4$ (ii). Therefore, it

\footnotetext{
${ }^{2}$ Argument as above.
} 
suffices to prove that $\bar{x} \in S(A ; b)=\operatorname{LSS}(A ; \hat{b})$. Let the index $i$ be arbitrary fixed in $[m]$. From $(* 21)$ it results that

$$
\forall k \geq 0, \exists s(k) \in\left\{k+1, \ldots, k+m_{0}\right\} \text { s.t. } i_{s(k)}=i,
$$

hence

$$
\left.\left.\left\langle A_{i_{s(k)}}, x^{s(k)-1}\right\rangle-b_{i_{s(k)}}^{s(k)}\right\rangle=\left\langle A_{i}, x^{s(k)-1}\right\rangle-b_{i}^{s(k)}\right\rangle \rightarrow 0 .
$$

From the inequalities in (15) it results that

$$
\left.\left.\left\langle A_{i}, x^{s(k)-1}\right\rangle-b_{i}\right\rangle \leq\left\langle A_{i}, x^{s(k)-1}\right\rangle-b_{i}^{s(k)}\right\rangle+\left|r_{i}-y_{i}^{s(k)}\right|,
$$

which together with (16) give us that $\left\langle A_{i}, \bar{x}\right\rangle=b_{i}$ and completes the proof. 